\title{
New signal processing for fast and accurate QEPAS measurements
}

\author{
Raphael Levy \\ DPHY \\ ONERA- The French Aerospace \\ Lab
}

Chatillon, France raphael.levy@onera.fr

\author{
Maxime Duquesnoy \\ DPHY \\ ONERA- The French Aerospace \\ $\mathrm{Lab}$ \\ Chatillon, France \\ maxime.duquesnoy@onera.fr
}

\author{
Guillaume Aoust \\ MIRSENSE
MIllaume Aoust \\ Palaiseau, France
}

\begin{abstract}
Quartz Enhanced Photoacoustic Spectroscopy (QEPAS) gas sensors have been widely developed over the last decade. This technique takes advantage of a high quality factor micro tuning fork to enable high detectivity \& high selectivity miniature gas sensors. Lock-in detection technique is classically used to measure the resonator amplitude proportional to gas concentration, but this technique is slow and does not follow the resonator frequency shift over temperature. This paper presents a new QEPAS signal processing technique that allows faster and more accurate measurements that will enable accurate and fast multi gas sensors.
\end{abstract}

Keywords-QEPAS, quartz, resonator, photoacoustic, spectroscopy

\section{INTRODUCTION}

QEPAS sensors have been widely studied since the first publication in 2002 [1]; models of the sensor have been developed [2,3] as well as optimized resonant detectors coupled to micro tubes acoustic resonator in different configurations [4] but little attention has been given to the signal processing scheme. Lock-in detection is mainly used although it shows major drawbacks: slow measurement and low accuracy, that prevent QEPAS to be used as rapid and accurate multi gas sensors.

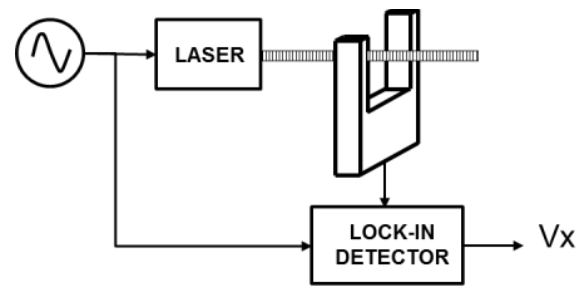

Indeed, in the frame of lock-in detection, the resonator is not controlled at resonance, and as the resonance shifts over temperature, a frequency sweep must be performed to detect the frequency that corresponds to the resonator maximum displacement. For each frequency point, the measurement time corresponds to :

$$
\tau=\frac{Q}{f_{r}}
$$

$\mathrm{Q}$ is the resonator quality factor, $\mathrm{f}_{\mathrm{r}}$ its Eigen frequency.
This measurement time can be long as $\mathrm{Q}$ must be high $(>10000)$ anf $\mathrm{f}_{\mathrm{r}}$ low $(<30 \mathrm{kHz})$ to achieve a high detectivity [3]. This long measurement time also decrease accuracy as measurements integrate the signal shift over temperature. It also prevent from high speed measurement of toxic gas and multi gas detection. The proposed active detection scheme enables faster and more accurate measurement, its theoretical analysis is presented as well as experimental measurements.

\section{THEORETICAL ANALYSIS OF QEPAS ACTIVE DETECTION}

\section{A. Principle of operation}

The principle of operation shown in figure 1 is as follows: the micro tuning fork is actuated at resonance by means of an oscillator circuit, the oscillator output signal is phase shifted and used to modulate the laser intensity. A phase quadrature is set between the resonator actuation force from the oscillator circuit and the photoacoustic actuation force, this induces a frequency shift of the oscillator that is proportional to the photoacoustic force and thus to the gas concentration. This frequency shift is instantaneous allowing fast measurements and the frequency variations over temperature can be cancelled by adding a $180^{\circ}$ periodic phase shift.

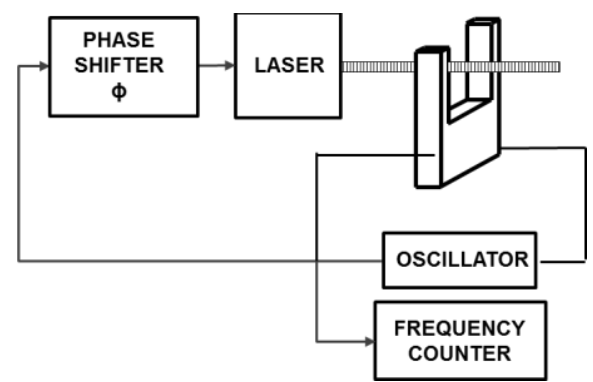

Fig. 1. QEPAS active detection scheme. The resonator oscillations are sustained by means of an oscillator circuit. The oscillator output signal is phase shifted and controls the modulation of the laser intensity. The measured frequency shift is proportional to the resonator vibration amplitude.

\section{B. Theoretical model}

The active detection scheme is presented in figure 2. Two feedback branches contribute to actuate the resonator: the oscillator circuit branch that leads to the piezoelectric actuation 
force $\mathrm{F}_{\mathrm{pz}}$, and the photoacoustic branch that leads to the photoacoustic force: $\mathrm{F}_{\mathrm{pa}}$. In order to obtain an oscillator frequency shift over the resonator displacement, these two forces must be in phase quadrature. This is performed by adjusting the phase shift $\Phi_{\text {elec }}$ through the phase shifter circuit. Indeed, the two modulated forces are in phase quadrature if:

$$
\frac{\pi}{2}=\Phi_{\text {pa }}+\Phi_{\text {propagation }}+\Phi_{\text {elec }}
$$

$\Phi_{p a}$ is the photoacoustic phase shift [5]:

$$
\Phi_{p a}=\frac{\pi}{2}-\operatorname{atan}\left(2 \pi f \tau_{V T}\right)
$$

$\mathrm{f}_{\mathrm{r}}$ is the modulation frequency, $\tau_{\mathrm{vt}}$ the $\mathrm{V}-\mathrm{T}$ relaxation time.

$\Phi_{\text {propagation }}$ is the acoustic propagation time from the laser beam to the resonator prongs:

$$
\Phi_{p a}=\frac{2 \pi \mathrm{L}}{\lambda}
$$

$\mathrm{L}$ is the distance from the beam to the resonator branch, $\lambda$ the modulation wavelength.

If the two actuation forces are in phase quadrature, the total force actuating the resonator is:

$$
\begin{gathered}
F_{\text {total }}=\sqrt{\left|F_{p z}\right|^{2}+\left|F_{p a}\right|^{2}} \sin \left(2 \pi f_{r} t+\theta\right) \\
\theta=\operatorname{Atan}\left(\frac{|F p a|}{|F x|}\right)
\end{gathered}
$$

This additional phase shift in the oscillator loop induces an oscillator frequency shift:

$$
\Delta f_{r}=\frac{\theta}{2 Q} f_{r}
$$

Assuming that the photoacoustic force is order og magnitudes smaller than the piezoelectric force:

$$
\Delta f_{r}=\frac{1}{2 Q} f_{r} \frac{|F p a|}{|F x|}
$$

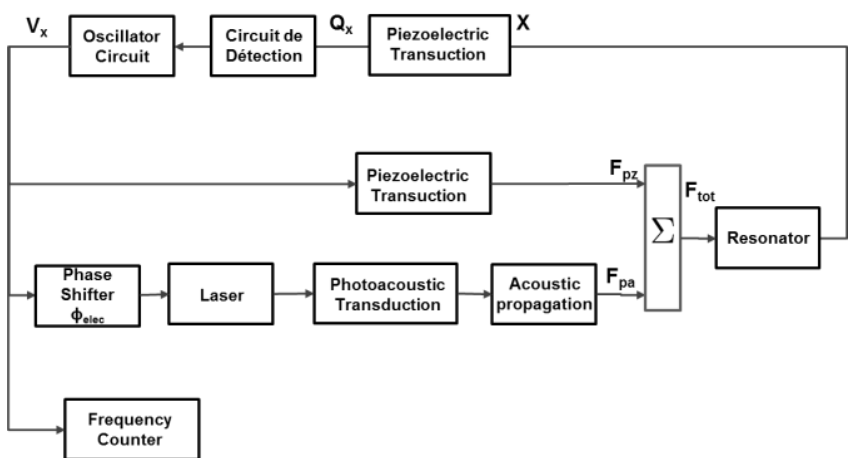

Fig. 2. Active detection scheme. The oscillator feedback circuit branch is presented as well as the photoacoustic feedback. The oscillator frequency shift, proportional to the resonator displacement, is measure by means of the frequency counter.

\section{Differential Measurements enabled}

The active detection scheme described above also makes it possible to overcome the frequency variations of the resonator over temperature by periodically adding a $\pi$ phase shift to the phase shifter circuit. Indeed a $\pi$ additional phase shift inverts the sign of the acoustic force $\mathrm{F}_{\mathrm{pa}}$ applied to the resonator, and thus inverts the sign of the phase shift $\theta$ induced in the oscillating loop (see eq. 6 ), which inverts the sign of the oscillator frequency variation (see eq. 7).

The frequency of the oscillator without supplementary phase shift is:

$$
\left.f_{r}\right|_{0}=f_{r 0}+\Delta f_{r}+\Delta f_{r}(T)
$$

The frequency of the oscillator with the $\pi$ supplementary phase shift is:

$$
\left.f_{r}\right|_{\pi}=f_{r 0}-\Delta f_{r}+\Delta f_{r}(T)
$$

$\Delta f_{r}(T)$ is the temperature induced frequency variation

The frequency variation over temperature is then cancelled by varying the additional phase shift between 0 and $\pi$ and subtracting the two measured frequencies:

$$
\left.f_{r}\right|_{0}-\left.f_{r}\right|_{\pi}=2 \Delta f_{r}
$$

The addition of a photoacoustic actuation force in phase quadrature with the piezoelectric force causes a phase shift in the oscillator loop. This phase shift must remain low enough for the oscillation conditions to remain valid. For this, the induced frequency variation must remain in the resonator bandwidth:

$$
\Delta f_{r} \ll \frac{f_{r}}{Q}
$$

\section{Noise calculation}

$$
\sigma_{O L}=\sqrt{4 k T \frac{M \omega_{r}}{Q} \frac{1}{\tau}}
$$

$$
\sigma C L=\frac{2 Q F P Z}{f r}\left(\sqrt{\frac{3 f H F k T R m}{4 \pi^{2} V x^{2}}} \frac{1}{\tau}+\sqrt{\frac{\ln (2) b_{-1}}{2}} \frac{f r}{Q}\right)
$$

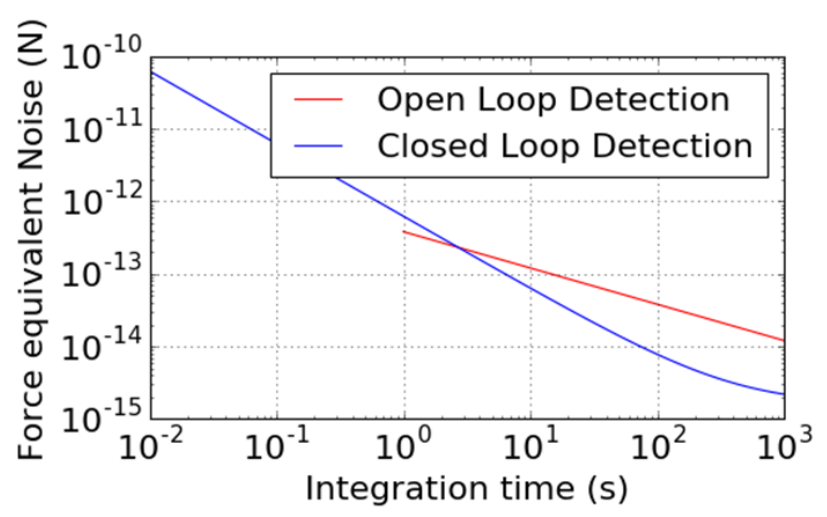

\section{EXPERIMENTAL RESULTS}

\section{A. Experimental set-up}

The experimental scheme is shown in figure 3 . The resonator is placed in a hermetic chamber filled with $1 \% \mathrm{C}_{2} \mathrm{H}_{2}$. 
The laser source is an EM4 diode, its wavenumber is set at the $\mathrm{C}_{2} \mathrm{H}_{2}$ absorption peak: $6490.05 \mathrm{~cm}^{-1}$. The oscillator circuit is an analog self-sustained oscillator, its output signal controls a waveform generator that enables to control the phase shift of the photoacoustic branch, as well as the waveform and the amplitude of the Mach Zender input. The oscillator frequency shift, proportional to the $\mathrm{C}_{2} \mathrm{H}_{2}$ concentration is measured with a frequency counter.

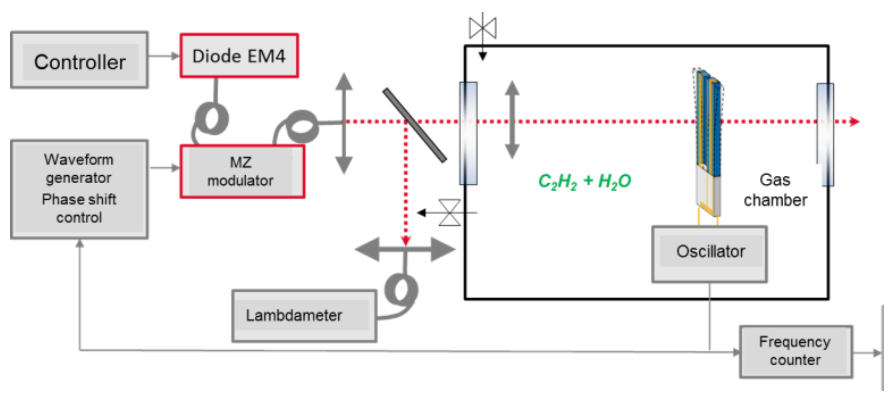

Fig. 3. Active detection experimental scheme

\section{B. Experimental results}

\section{1) Setting the phase shift}

\section{2) Frequency shift measurement}

Figure 5 shows the oscillator frequency variations with and without a $\pi$ additional phase shift. When the laser is on, the frequency increases when the additional phase shift is null, and decreases when the additional phase shift is $\pi$, as described in eq. 9 and 10. An additional frequency decrease over time is common to the two oscillator frequencies; it corresponds to the frequency variation over temperature. This temperature sensitivity can be cancelled by computing the difference of the two frequencies, as shown in figure 6 and described in eq. 11.

The measurement time used in figure 4 and 5 is $1 \mathrm{~s}$, measurements have also been performed down to $50 \mathrm{~ms}$ in order to validate the fast measurements ability of the active detection.

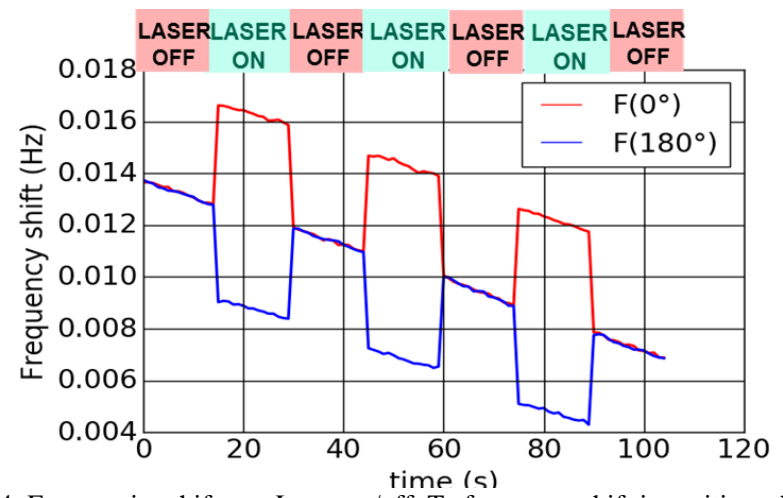

Fig. 4. Frequencies shifts vs. Laser on/off. Te frequency shift is positive when the additional phase dhift is null (red line), whereas it is negative when the additional phase shift is $180^{\circ}$ (blue line). In addition to the photoacoustic signal frequency sensitivity, the frequency also shift over time due to temperature variations.

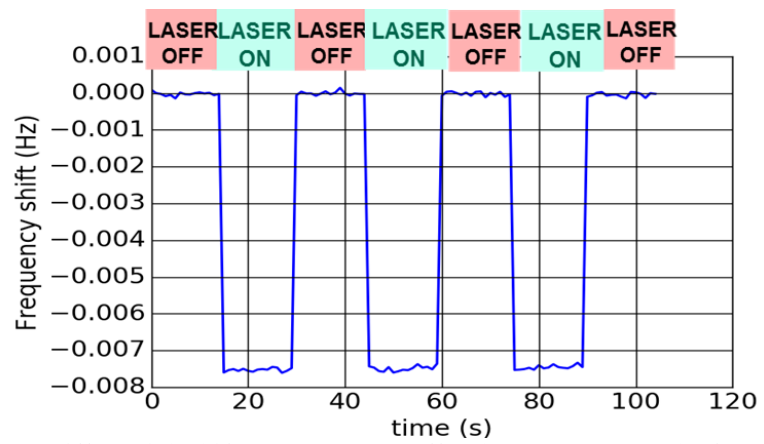

Fig. 5. Differential shift vs. Laser on/off. The common mode frequency variation over temperature has been cancelled by the differential measurement.

\section{CONCLUSION}

A new active detection scheme has been described; a theoretical model has been shown and validated by experimental measurements. This new detection scheme enables fast and accurate measurements. Fast measurements down to $50 \mathrm{~ms}$ integration time have been performed and confirm the accurate and thermal drift free measurements. Further work should focus on the signal to noise ratio improvements by optimizing the resonator and its electrodes scheme, and by implementing the detection scheme in synchronous digital electronics on FPGA.

[1] Kosterev, Anatoliy A., et al. "Quartz-enhanced photoacoustic spectroscopy." Optics letters 27.21 (2002): 1902-1904.

[2] Petra, N., Zweck, J., Kosterev, A. A., Minkoff, S. E., \& Thomazy, D. (2009). Theoretical analysis of a quartz-enhanced photoacoustic spectroscopy sensor. Applied Physics B, 94(4), 673-680.

[3] Aoust, G., Levy, R., Raybaut, M., Godard, A., Melkonian, J. M., \& Lefebvre, M. (2017). Theoretical analysis of a resonant quartz-enhanced photoacoustic spectroscopy sensor. Applied Physics B, 123(2), 63.

[4] Patimisco, P., Scamarcio, G., Tittel, F. K., \& Spagnolo, V. (2014). Quartz-enhanced photoacoustic spectroscopy: a review. Sensors, 14(4), 6165-6206.

[5] Cottrell, T. L., \& McCoubrey, J. C. (1961). Molecular energy transfer in gases. Butterworths. 